\title{
碳化多孔有机骨架制备氮掺杂多孔碳及其气体吸附研究
}

\author{
李艳强 ${ }^{a}$ 贲腾 $*, b$ 装式纶*, $a$ \\ ( ${ }^{a}$ 吉林大学无机合成与制备化学国家重点实验室＼cjkstart长春 130012) \\ ${ }^{b}$ 吉林大学化学学院 长春 130012)
}

\begin{abstract}
摘要 通过简单的一步碳化方法, 以含氮的多孔有机骨架 JUC-Z2 为碳前驱物制备出氮掺杂多孔碳材料. 与原始 JUC-Z2 材料相比, 制备的多孔碳材料显示出明显提高的气体吸附量和增强的吸附焓. 其中 JUC-Z2-900 的 $\mathrm{CO}_{2}$ 吸附量 高达 $113 \mathrm{~cm}^{3} \cdot \mathrm{g}^{-1}, \mathrm{H}_{2}$ 吸附量也达到 $246 \mathrm{~cm}^{3} \cdot \mathrm{g}^{-1}$, 超过了大部分报道的多孔材料. 尤其是 JUC-Z2-900 的 $\mathrm{CH}_{4}$ 吸附量在 $273 \mathrm{~K}, 1 \mathrm{bar}$ 下高达 $60 \mathrm{~cm}^{3} \cdot \mathrm{g}^{-1}$, 据我们所知, 这一值为目前报道材料的最高值. 除此之外, 样品还显示出选择性吸附 $\mathrm{CO}_{2}$ 的能力, $273 \mathrm{~K}$ 下, JUC-Z2-900 的 $\mathrm{CO}_{2} / \mathrm{N}_{2}$ 的选择性高达 $10, \mathrm{CO}_{2} / \mathrm{H}_{2}$ 的选择性也高达 66 . 另外, 样品具有很高的热稳 定性, 有望应用在碳捕获和清洁能源储存等领域.
\end{abstract}

关键词 多孔有机骨架; 多孔碳; $\mathrm{CO}_{2}$ 吸附; 储氢; 选择性吸附

\section{Preparation of N-doped Porous Carbon from Porous Organic Framework for Gas Sorption}

\author{
Li, Yanqiang $^{a} \quad$ Ben, Teng*, $\quad$ Qiu, Shilun*,a \\ ( ${ }^{a}$ State Key Laboratory of Inorganic Synthesis \& Preparation Chemistry, Jilin University, Changchun 130012) \\ ( ${ }^{b}$ College of Chemistry, Jilin University, Changchun 130012)
}

\begin{abstract}
In this report, a series of N-doped porous carbon materials were successfully prepared from nitrogen-containing porous organic framework JUC-Z2. Compared to original JUC-Z2, the carbonized samples show obviously enhanced gas uptake and isosteric heats of adsorption ( $Q_{\text {st }}$ for short). Among the carbonized samples, JUC-Z2-900 shows high $\mathrm{CO}_{2}$ uptake of $113 \mathrm{~cm}^{3} \cdot \mathrm{g}^{-1}$ at $273 \mathrm{~K}$ and $1 \mathrm{bar}$ and $\mathrm{H}_{2}$ sorption of $246 \mathrm{~cm}^{3} \cdot \mathrm{g}^{-1}$ at $77 \mathrm{~K}$ and $1 \mathrm{bar}$, surpassing most reported porous materials. Especially for $\mathrm{CH}_{4}$ sorption, a large sorption amount of $60 \mathrm{~cm}^{3} \cdot \mathrm{g}^{-1}$ could be achieved at $273 \mathrm{~K}$ and 1 bar. To our best knowledge, this value is comparable to the highest among all the porous materials reported to date. Apart from high gas uptake, the carbon materials also show selective adsorption ability. At $273 \mathrm{~K}$, JUC-Z2-900 shows a high $\mathrm{CO}_{2} / \mathrm{N}_{2}$ adsorption selectivity of 10 and $\mathrm{CO}_{2} / \mathrm{H}_{2}$ adsorption selectivity of 66 . Raman spectra showed two Raman shifts, the G-band at $1590 \mathrm{~cm}^{-1}$ is associated with the $\mathrm{E}_{2 \mathrm{~g}}$ mode of graphite, whereas the D-band centered at around $1360 \mathrm{~cm}^{-1}$ is attributed to the D-band of disordered carbon, corresponding to the defect-induced mode. The intensity of D-band is higher than G-band, indicating a low degree of graphitization. This is also confirmed by powder X-ray diffraction results. X-ray Photoelectron Spectroscopy (XPS) results indicate the nitrogen content is $3.26 \mathrm{wt} \%, 2.88 \mathrm{wt} \%$ and $2.19 \mathrm{wt} \%$ for JUC-Z2-700, JUC-Z2-800 and JUC-Z2-900 respectively. Though the nitrogen content decreased after carbonization, the gas sorption increased greatly. This can be attributed to the increased heat of adsorption of the carbonized samples. First, the narrow pore size after carbonization is beneficial for gas storage. Reports indicate that by tuning the pore sizes to around the kinetic diameter of $\mathrm{CO}_{2}$, it may be possible to increase the number of double or multiple interactions between the adsorbed $\mathrm{CO}_{2}$ and the pore walls. Second, the all-carbon-scaffold networks also benefit the gas-adsorbent interaction. Last but not the least, the N-doped framework also devote the high gas uptake. Besides the high gas uptake, the carbon materials exhibit high thermal stabilities and could be stable up to $500{ }^{\circ} \mathrm{C}$. Based on the above results, the carbon materials show great potential in the fields of $\mathrm{CO}_{2}$ capture and clean energy storage.

Keywords porous organic frameworks; porous carbons; $\mathrm{CO}_{2}$ sorption; $\mathrm{H}_{2}$ storage; selective adsorption
\end{abstract}

\section{1 引言}

\section{化石燃料燃烧排放的 $\mathrm{CO}_{2}$ 引起了一系列的全球性}

环境问题例如全球气候变暖、冰川融化、海平面上升、 海水酸性增加及气候异常等现象, 严重威胁着人类的生

\footnotetext{
*E-mail: sqiu@jlu.edu.cn; tben@jlu.edu.cn; Tel.: 0086-0431-85168589

Received January 31, 2015; published April 23, 2015.

Supporting information for this article is available free of charge via the Internet at http://sioc-journal.cn

Project supported by National Natural Science Foundation of China (Grant no. 21390394, 21261130584, 21471065), the National Basic Research Program of China (2012CB821700), the "111" project (B07016), the Award Project of KAUST (CRG-1-2012-LAI-009) and the Ministry of Education, Science and Technology Development Center Project (20120061130012).

项目受国家自然科学基金(21390394，21261130584，21471065)，国家基础研究计划(2012CB821700)，“111”计划(B07016), KAUST 奖励项目 (CRG-1-2012-LAI-009)和教育部科技发展中心项目(20120061130012)资助.
} 
存与发展, 使得人们急于寻求降低大气中 $\mathrm{CO}_{2}$ 气体含量 的方法. 碳的捕集和封存 (carbon capture and storage, $\mathrm{CCS})^{[1]}$ 是科学家们提出的减少二氧化碳排放, 防止气候 恶化的技术方案之一. 传统的捕获 $\mathrm{CO}_{2}$ 的方法是利用胺 溶液, 如发电厂中利用单乙醇胺从尾气中捕获 $\mathrm{CO}_{2}{ }^{[2]}$. 虽然这种方法具有吸收速度快吸附能力大等优点, 但是 胺溶液的再生需要消耗很高的能量并且胺溶液会引起 设备腐蚀 ${ }^{[3]}$. 因此寻求有效捕获 $\mathrm{CO}_{2}$ 的材料是目前的一 个研究热点. 另一个降低大气中 $\mathrm{CO}_{2}$ 含量的方法是使用 清洁能源. $\mathrm{H}_{2}$ 作为清洁高效的能源备受人们的关注, 其 具有取之不尽用之不竭的来源一一水, 并且燃烧排放零 污染 $^{[4]}$. 但是在氢气作为主要能源的时代来临之前, 还 有很多技术问题需要解决, 其中一个关键步骤就是 $\mathrm{H}_{2}$ 运输. 通常可以将 $\mathrm{H}_{2}$ 压缩成液态的形式存储在高压罐 里, 但是此过程需要消耗大量能量, 并且存在巨大的安 全隐患. 甲烷作为天然气的主要成分, 是烃类化合物中 氢碳比最高的化合物, 也是清洁能源的备选, 但是其储 存面临同样问题. 因此急需发展安全高效的清洁能源储 存方法.

近年来多孔有机骨架化合物作为一种新型的多孔 材料受到人们的广泛关注, 其具有高的表面积, 开放的 骨架, 多样的结构, 在气体吸附、分子识别、催化以及 光、电等领域有着广泛的应用前景 ${ }^{[5 \sim 17]}$. 其中, 多孔芳 香骨架, 由于其超高的比表面积和优异的物理化学稳定 性等特点, 在气体吸附领域显示出明显优势 ${ }^{[18 ~ 20]}$. 例如 多孔芳香骨架 PAF-1 具有高达 $5640 \mathrm{~m}^{2} \cdot \mathrm{g}^{-1}$ 的比表面积 和均匀的微孔分布, 高压下对氢气(质量分数超过 $7.0 \%$, $77 \mathrm{~K} / 48 \mathrm{bar}$ )和二氧化碳(1.3 g・g $\left.{ }^{-1}, 298 \mathrm{~K} / 40 \mathrm{bar}\right)$ 具有良 好的吸附性 ${ }^{[18]}$. 但是由于其空旷的骨架, 与气体分子之 间相互作用较弱，使得其在低压下的气体吸附性质不够 理想. 很多种方法可以用来对其加以改性以提高其与气 体分子之间的相互作用, 从而提高其低压下的气体吸附 量, 如磺酸化, 胺接枝, 锂代等 ${ }^{[21 ~ 23]}$. 我们组通过对其 进行高温热处理, 发现可以有效地调节其孔道大小, 从 而提高骨架与气体分子之间相互作用, 得到吸附性质明 显提高的材料 ${ }^{[24]}$. 最近的研究显示, 向多孔材料骨架中 引入 $\mathrm{N}, \mathrm{B}, \mathrm{S}$ 等原子可以有效地提高与气体的相互作用 位点, 从而提高气体吸附量 ${ }^{[25 ~ 27]}$. 多种杂原子掺杂的多 孔碳被报道, 显示出提高的气体吸附能力. 本文选用含 氮多孔有机骨架 JUC-Z2 $2^{[28]}$ 作为碳源, 制备氮原子掺杂 的多孔碳. 结果显示碳化之后, JUC-Z2 骨架中的氮原子 能够有效的得以保留, 同时得到的多孔碳具有窄的微孔 分布和较高的比表面积, 显示出提高的气体吸附能力.

\section{2 结果与讨论}

\section{1 结构表征}

JUC-Z2 通过镍催化的 Yamamoto type Ullmann 交叉 偶联反应合成, 是利用三(四溴苯基)胺为单体, 双-(1,5-
环辛二烯)镍为催化剂, 经过脱溴偶联而成的 $h c b$ 拓扑的 聚三苯基胺结构, 详细的合成路线见支持信息(图 S1). 得到的白色粉末具有高达 $2076 \mathrm{~m}^{2} \cdot \mathrm{g}^{-1}$ 的表面积(Table $\mathrm{S} 1$ ), 和文献中报道结果一致 ${ }^{[28]}$. 将 JUC-Z2 在氮气保护 下进行碳化, 得到的样品命名为 JUC-Z2-X, 其中 X 代 表碳化温度. 图 $1 \mathrm{~A}$ 是所得样品的拉曼光谱, 可以看到 所有的样品均显示出 $1590 \mathrm{~cm}^{-1}$ 对应于石墨碳的 $\mathrm{G}$ 带和 $1360 \mathrm{~cm}^{-1}$ 对应于无序碳的 D 带. D 带的强度要大于 $G$ 带, 表明石墨化度较低, 一方面由于材料较高的多孔性 引起有序度的下降, 另一方面氮原子掺杂也引起 $\mathrm{D}$ 带的 增强. X 射线粉末衍射也显示材料较低的石墨化度. $24^{\circ}$ 和 $44^{\circ}$ 为中心的两个衍射峰对应于碳材料(002)和(100) 衍射峰(图 S2). 通过XPS 测试多孔碳材料中氮元素的含 量(图 1B), JUC-Z2-700, JUC-Z2-800 和 JUC-Z2-900 中氮 原子百分含量分别为 $3.26 \mathrm{wt} \%, 2.88 \mathrm{wt} \%, 2.19 \mathrm{wt} \%$, 均
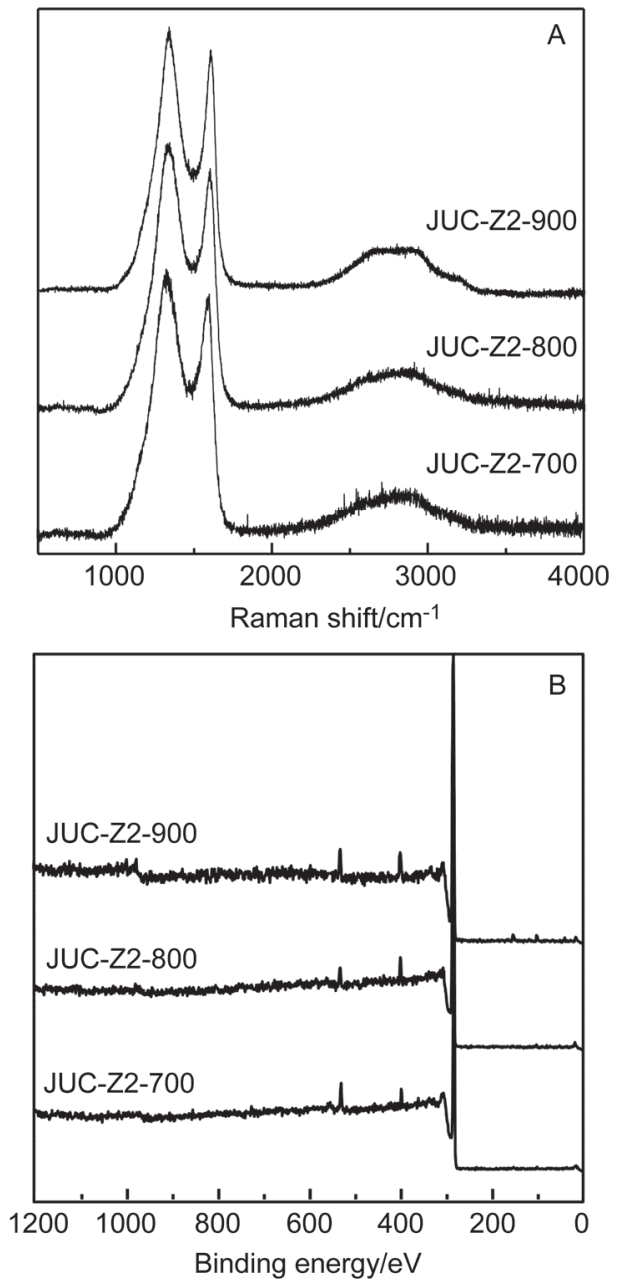

图 1 (A)碳化样品 JUC-Z2-700, JUC-Z2-800 和 JUC-Z2-900 的拉曼光 谱; (B)碳化样品 JUC-Z2-700, JUC-Z2-800 和 JUC-Z2-900 的 X 射线光 电子能谱

Figure 1 (A) Raman spectra of the carbonized samples JUC-Z2-700, JUC-Z2-800 and JUC-Z2-900; (B) XPS spectra of the carbonized samples JUC-Z2-700, JUC-Z2-800 and JUC-Z2-900 
低于原料 JUC-Z2 中的氮含量 $(5 \mathrm{wt} \%)$, 且随着碳化温度 的升高含量下降, 表明高温下氮物种的不稳定性, 这一 结果与文献中报道其它氮掺杂多孔碳结果一致 ${ }^{[29,30]}$. XPS 的 N1S 结果表明氮元素在材料中有石墨化氮和吡 啶型氮两种形式，吡啶型氮的存在表明多孔碳中继承了 一些多孔有机骨架前驱物的结构, 而石墨化氮可能来自 于高温下生成的石墨化结构(图 S3). 元素分析结果也显 示 JUC-Z2-700, JUC-Z2-800 和 JUC-Z2-900 中氮原子含 量分别为 $3.14 \mathrm{wt} \%, 2.72 \mathrm{wt} \%$ 和 $2.11 \mathrm{wt} \%$, 与 XPS 的测 试结果基本一致. 热重测试显示材料具有非常好的热稳 定性, 在空气中可以稳定在 $500{ }^{\circ} \mathrm{C}$ (图 S4).

\section{2 多孔性表征}

通过 $77 \mathrm{~K}$ 下的氮气吸附研究材料的多孔性. 所有 样品的氮气吸附曲线在低压范围内陡峭上升, 表明材料 的微孔特性. JUC-Z2 脱附曲线与吸附曲线存在一个迟 滞环, 而碳化后样品均显示出完全可逆的 I 型吸附曲线, 表明 JUC-Z2 骨架中原本存在的柔性部分已经消失, 转 化为全刚性的碳骨架(图 2A). 碳化之后由于骨架坍塌和 孔收缩, 样品的比表面迅速下降, JUC-Z2-700 的比表面 积仅为 $643 \mathrm{~m}^{2} \cdot \mathrm{g}^{-1}$, 而随着温度升高, 样品的比表面积 又逐渐增大, 可能是由于高温下骨架重排. JUC-Z2-800 和 JUC-Z2-900 的比表面积为 707 和 $805 \mathrm{~m}^{2} \cdot \mathrm{g}^{-1}$. 碳化引 起的另一个显著变化是孔尺寸收缩. 通过非定域密度泛 函理论(nonlocal density functional theory, NLDFT)计算 样品的孔分布. JUC-Z2 的孔尺寸为 $1.18 \mathrm{~nm}$ (图 S5), 而 碳化后的样品孔尺寸均显著收缩到 $0.6 \mathrm{~nm}$ 左右(图 2B). 孔体积也由 JUC-Z2 的 $1.09 \mathrm{~cm}^{3} \cdot \mathrm{g}^{-1}$ 下降为 $0.4 \mathrm{~cm}^{3} \cdot \mathrm{g}^{-1}$ 左右, 需要注意的是尽管总孔体积明显下降, 但是微孔 孔体积下降并不显著, 尤其是 JUC-Z2-900, 仍然具有高 达 $0.31 \mathrm{~cm}^{3} \cdot \mathrm{g}^{-1}$ 的微孔孔体积, 而这些微孔孔体积对于 低压下气体吸附十分有利。

\section{3 气体吸附性质}

首先我们测试了碳化后样品对于温室气体 $\mathrm{CO}_{2}$ 的 低压吸附, 测试在 $273 \mathrm{~K}$ 和 $298 \mathrm{~K}$ 下进行. 如图 $3 \mathrm{~A}$ 所示 是样品在 $273 \mathrm{~K}$ 下的 $\mathrm{CO}_{2}$ 吸附曲线. 尽管碳化之后样品 的比表面积有所下降, 但是可以看出碳化后的样品均显 示出提高的 $\mathrm{CO}_{2}$ 吸附量. JUC-Z2 在 1 bar 下 $\mathrm{CO}_{2}$ 吸附量 为 $71 \mathrm{~cm}^{3} \cdot \mathrm{g}^{-1}$, 而碳化后的样品 JUC-Z2-700, JUC-Z2-800 和 JUC-Z2-900 这一值分别为 80, 96 和 113 $\mathrm{cm}^{3} \cdot \mathrm{g}^{-1}$. 并且吸附曲线完全可逆且没有达到饱和, 也就 是说随着测试压强增大样品将进一步吸附更多的 $\mathrm{CO}_{2}$. 为了研究 $\mathrm{CO}_{2}$ 吸附量显著提高的原因, 我们利用 Clausius-Clapeyron 方程计算了样品的吸附焓, 如图 3B 所示. 可以看出样品的吸附焓有明显的增加, 相对于 $\mathrm{JUC}-\mathrm{Z2}$ 的 $23 \mathrm{~kJ} \cdot \mathrm{mol}^{-1}$, JUC-Z2-700, JUC-Z2-800 和 JUC-Z2-900 的 $\mathrm{CO}_{2}$ 吸附焓分别为 $30.2,30.4$ 和 25.4
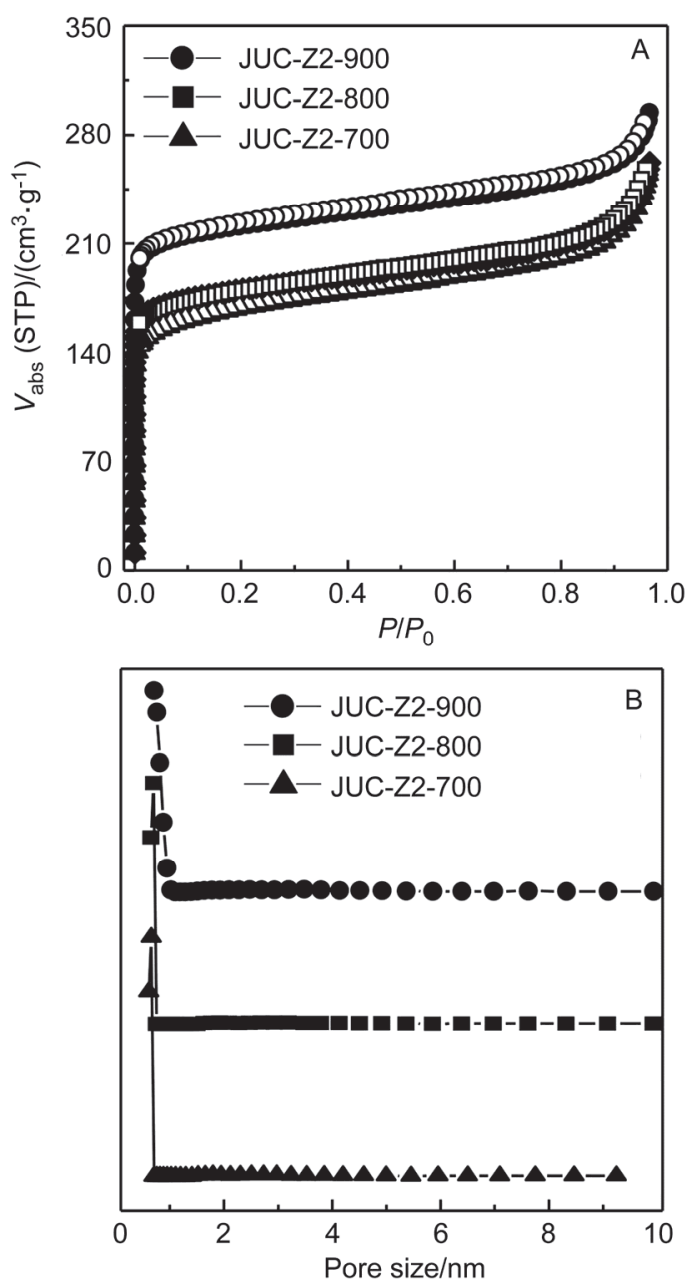

图 2 (A)碳化样品 JUC-Z2-700, JUC-Z2-800 和 JUC-Z2-900 的 $\mathrm{N}_{2}$ 吸 附-脱附等温线(实心为吸附曲线, 空心为脱附曲线); (B)由吸附曲线 通过非定域密度泛函理论计算的样品的孔分布曲线

Figure 2 (A) $\mathrm{N}_{2}$ sorption isotherms (solid symbols, adsorption; open symbols, desorption) and (B) pore size distributions of JUC-Z2-700, JUC-Z2-800 and JUC-Z2-900 derived from $\mathrm{N}_{2}$ adsorption calculated by nonlocal density functional theory

$\mathrm{kJ} \cdot \mathrm{mol}^{-1}$. 尤其 JUC-Z2-700 和 JUC-Z2-800, $\mathrm{CO}_{2}$ 吸附焓 提高了 $30 \%$ 之多. 样品提高的吸附焓可能来源于以下几 种原因: 首先, 碳化之后样品孔径大幅下降增强了吸附 质一吸附剂相互作用. 据报道, 调节孔尺寸在 $\mathrm{CO}_{2}$ 分子 的动力学直径附近, 可以增强 $\mathrm{CO}_{2}$ 与孔壁之间 $2 \sim 3$ 倍 相互作用力. 其次, 碳化后得到的纯碳骨架与具有较大 四极距的 $\mathrm{CO}_{2}$ 分子之间将有更强的亲和力. 最后, 样品 中的碱性的氮原子与酸性 $\mathrm{CO}_{2}$ 分子之间强的相互作用 也有利于吸附焓增加, 如不含杂原子的多孔芳香骨架

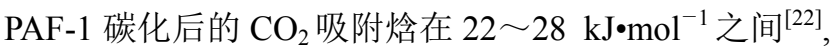
要低于我们这里的报道值. 同时 JUC-Z2-900 相对于 JUC-Z2-700 和 JUC-Z2-800 吸附焓略低也可能是由于碳 化温度较高引起氮含量下降的原因. 

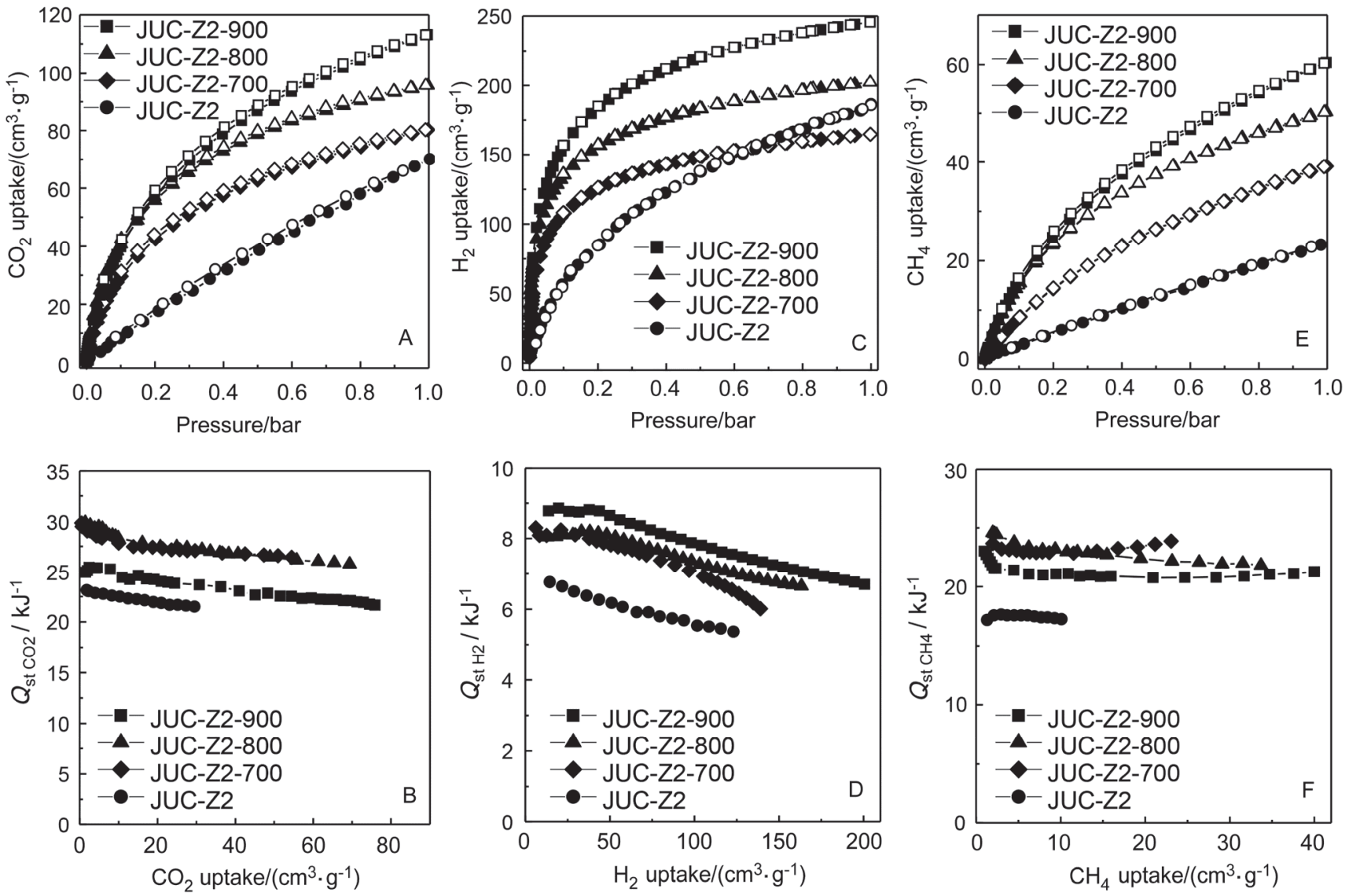

图 3 (A) $273 \mathrm{~K}$ 下 JUC-Z2, JUC-Z2-700, JUC-Z2-800 和 JUC-Z2-900 的低压 $\mathrm{CO}_{2}$ 吸附-脱附等温线(实心为吸附曲线, 空心为脱附曲线); (B) JUC-Z2, JUC-Z2-700, JUC-Z2-800 和 JUC-Z2-900 的 $\mathrm{CO}_{2}$ 吸附焓曲线; (C) $77 \mathrm{~K}$ 下 JUC-Z2, JUC-Z2-700, JUC-Z2-800 和 JUC-Z2-900 的低压 $\mathrm{H}_{2}$ 吸附-脱附等 温线(实心为吸附曲线, 空心为脱附曲线); (D) JUC-Z2, JUC-Z2-700, JUC-Z2-800 和 JUC-Z2-900 的 $\mathrm{H}_{2}$ 吸附焓曲线; (E) $273 \mathrm{~K}$ 下 JUC-Z2, JUC-Z2-700, JUC-Z2-800 和 JUC-Z2-900 的低压 $\mathrm{CH}_{4}$ 吸附-脱附等温线(实心为吸附曲线, 空心为脱附曲线); (F) JUC-Z2, JUC-Z2-700, JUC-Z2-800 和 JUC-Z2-900 的 $\mathrm{CH}_{4}$ 吸附焓曲线

Figure 3 (A) $\mathrm{CO}_{2}$ adsorption (solid symbols) and desorption (open symbols) isotherms of JUC-Z2 and carbonized samples at $273 \mathrm{~K}$; (B) $Q_{\mathrm{stCO} 2}$ of JUC-Z2 and carbonized samples as a function of the amount of $\mathrm{CO}_{2}$ adsorbed. (C) $\mathrm{H}_{2}$ adsorption (solid symbols) and desorption (open symbols) isotherms of JUC-Z2 and carbonized samples at $77 \mathrm{~K}$; (D) $Q_{\mathrm{stH} 2}$ of JUC-Z2 and carbonized samples as a function of the amount of $\mathrm{H}_{2}$ adsorbed. (E) $\mathrm{CH}_{4}$ adsorption (solid symbols) and desorption (open symbols) isotherms of JUC-Z2 and carbonized samples at $273 \mathrm{~K}$; (F) $Q_{\text {stCH4 }}$ of JUC-Z2 and carbonized samples as a function of the amount of $\mathrm{CH}_{4}$ adsorbed

氢气作为高能量密度的清洁能源，被认为是替代化 石燃料的优良备选. 如果能将 $\mathrm{H}_{2}$ 有效地存储在固体吸 附剂中，就能解决 $\mathrm{H}_{2}$ 储存和运输中的安全性问题. 基于 上述目的，我们测试了碳化后样品的 $\mathrm{H}_{2}$ 储存性质. 如图 $3 \mathrm{C}$ 所示是样品在 $77 \mathrm{~K}$ 下的低压 $\mathrm{H}_{2}$ 吸附曲线. 可以看出 吸附曲线完全可逆没有迟滞环, 表明物理吸附机理. 相 对于 JUC-Z2 的吸附量 $181 \mathrm{~cm}^{3} \cdot \mathrm{g}^{-1}$ 而言, JUC-Z2-700 的 $\mathrm{H}_{2}$ 吸附量略有下降, 为 $165 \mathrm{~cm}^{3} \cdot \mathrm{g}^{-1}$, 可能是由于其比表 面大幅下降的原因. JUC-Z2-800 和 JUC-Z2-900 显示出 明显提高的 $\mathrm{H}_{2}$ 吸附量, 分别为 201 和 $246 \mathrm{~cm}^{3} \cdot \mathrm{g}^{-1}$. 尤其 是, 在 0.7 个大气压之前碳化样品的吸附量均高于 JUC-Z2, 这对于较低浓度下 $\mathrm{H}_{2}$ 吸附十分有效. 碳化后 的样品均显示出明显高于 JUC-Z2 的 $\mathrm{H}_{2}$ 吸附焓, 在 $8.2 \sim 8.8 \mathrm{~kJ}^{\circ} \mathrm{mol}^{-1}$ 之间, 而 JUC-Z2 仅为 $6.8 \mathrm{~kJ} \cdot \mathrm{mol}^{-1}$, 具 体结果见表 1 . 明显提高的吸附焓也可能来自于碳化后
样品窄的孔分布和氮原子掺杂. 需要指出的是 JUC-Z2-900 的 $\mathrm{H}_{2}$ 吸附量 $246 \mathrm{~cm}^{3} \cdot \mathrm{g}^{-1}$ 超过了同样条件下 很多多孔材料如金属有机骨架, 共价有机骨架, 多孔聚 合物, 多孔碳等 ${ }^{[31 ~ 33]}$.

烃类化合物中氢碳比最高的化合物一一甲烷，是天 然气的主要成分，也是一种高效、污染小的清洁能源. 但是甲烷液化增加了其在储存运输时的成本，因此如何 高效的廉价的储存甲烷也是天然气利用过程中需要解 决的一个关键问题. 最近多孔材料在储存甲烷中显示出 巨大潜能，如金属有机骨架 PCN-14 甲烷储存能力已经 超出美国能源部的规定目标 ${ }^{[34,35]}$. 但这一测试是在高压 条件下测试，而对于从堆填区沼气(landfill gas)中吸附 $\mathrm{CH}_{4}$ 却需要在低压下进行. $273 \mathrm{~K}$ 下, JUC-Z2 低压下 $\mathrm{CH}_{4}$ 吸附量为 $25 \mathrm{~cm}^{3} \cdot \mathrm{g}^{-1}$, 尽管已经超过相同条件下的其它 多孔有机骨架如 PAF-1 ${ }^{[19]}$, 但是低的吸附量仍然限制了 
表 1 JUC-Z2 及碳化样品的气体吸附量和吸附焓总结

Table 1 Summary of $\mathrm{CO}_{2}, \mathrm{CH}_{4}, \mathrm{H}_{2}$ storage capacities and heats of adsorption of JUC-Z2 and carbonized samples

\begin{tabular}{|c|c|c|c|c|c|c|}
\hline \multirow[b]{2}{*}{ Samples } & \multicolumn{6}{|c|}{ Gas uptakes } \\
\hline & $\begin{array}{c}\mathrm{CO}_{2} \\
\left(\mathrm{~cm}^{3} \cdot \mathrm{g}^{-1}\right)\end{array}$ & $\begin{array}{c}Q_{\text {st CO2 }} \\
\left(\mathrm{kJ} \cdot \mathrm{mol}^{-1}\right)\end{array}$ & $\begin{array}{c}\mathrm{H}_{2} \\
\left(\mathrm{~cm}^{3} \cdot \mathrm{g}^{-1}\right)\end{array}$ & $\begin{array}{c}Q_{\text {st H2 }} \\
\left(\mathrm{kJ} \cdot \mathrm{mol}^{-1}\right)\end{array}$ & $\begin{array}{c}\mathrm{CH}_{4} \\
\left(\mathrm{~cm}^{3} \cdot \mathrm{g}^{-1}\right)\end{array}$ & $\begin{array}{c}Q_{\text {st CH4 }} \\
\left(\mathrm{kJ} \bullet \mathrm{mol}^{-1}\right)\end{array}$ \\
\hline JUC-Z2 & 71 & 23 & 181 & 6.8 & 25 & 14.0 \\
\hline JUC-Z2-700 & 80 & 30.2 & 165 & 8.2 & 39 & 24.4 \\
\hline JUC-Z2-800 & 96 & 30.4 & 202 & 8.3 & 50 & 25.0 \\
\hline JUC-Z2-900 & 113 & 25.4 & 246 & 8.8 & 60 & 24.0 \\
\hline
\end{tabular}

它的应用。碳化后的 JUC-Z2-700, JUC-Z2-800 和 JUC-Z2-900 甲烷吸附量显著提高, 分别为 $39 \mathrm{~cm}^{3} \cdot \mathrm{g}^{-1}$, $50 \mathrm{~cm}^{3} \cdot \mathrm{g}^{-1}$ 和 $60 \mathrm{~cm}^{3} \cdot \mathrm{g}^{-1}$. 尤其是 JUC-Z2-900, 据我们 所知, 这一值是目前报道的多孔材料 $\mathrm{CH}_{4}$ 吸附量最大 值. $\mathrm{CH}_{4}$ 吸附焓也由 JUC-Z2 的 $14 \mathrm{~kJ} \bullet \mathrm{mol}^{-1}$ 上升到 24 $\mathrm{kJ}^{\circ} \mathrm{mol}^{-1}$ 左右, 显示出材料明显提高的吸附能力.

在实际应用上, $\mathrm{CO}_{2}$ 的吸附往往不是在纯的 $\mathrm{CO}_{2}$ 环 境下进行, 而是在包含很多种气体的混合气体中吸附, 因此评价一个吸附 $\mathrm{CO}_{2}$ 材料时, 不仅需要考虑吸附量的 大小, 从其它竞争成分中选择性吸附 $\mathrm{CO}_{2}$ 能力也至关重 要. 人们为了评价材料的选择性吸附能力, 提出多种理 论方法, 其中使用较为广泛的有以下三种: 用纯组分气 体在一定压强下吸附量直接相除的方法; 亨利定律 (Henry's law)即吸附曲线在低覆盖下的斜率进行计算; 理想吸附溶液理论 (IAST), 这里我们分别测试了 JUC-Z2-900 在 $273 \mathrm{~K}$ 下的 $\mathrm{CO}_{2}, \mathrm{CH}_{4}, \mathrm{H}_{2}, \mathrm{~N}_{2}$ 吸附曲线, 如图 4 所示. 可以看出, 同样条件下, JUC-Z2-900 显示 出很高的 $\mathrm{CO}_{2}$ 和 $\mathrm{CH}_{4}$ 吸附量, 而对于 $\mathrm{N}_{2}$ 和 $\mathrm{H}_{2}$ 吸附量很 少, 显示出材料突出的选择性吸附能力. 利用第一种方 法即同温同压下纯组分气体吸附量直接相除来计算 JUC-Z2-900 的选择性吸附能力, $\mathrm{CO}_{2} / \mathrm{N}_{2}$ 选择性为 10 , $\mathrm{CO}_{2} / \mathrm{H}_{2}$ 选择性为 66 , 显示出样品潜在的 $\mathrm{CO}_{2}$ 选择性吸 附能力.

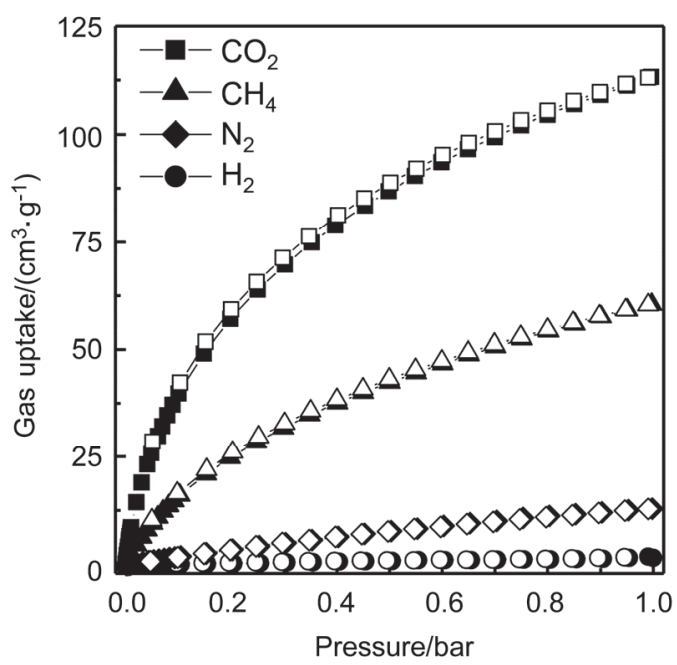

图 $4273 \mathrm{~K}$ 下 JUC-Z2-900 的 $\mathrm{CO}_{2}, \mathrm{CH}_{4}, \mathrm{~N}_{2}$ 和 $\mathrm{H}_{2}$ 吸附曲线 Figure $4 \mathrm{CO}_{2}, \mathrm{CH}_{4}, \mathrm{~N}_{2}$ and $\mathrm{H}_{2}$ sorption of JUC-Z2-900 at $273 \mathrm{~K}$

\section{3 结论}

通过简单的一步碳化含氮的多孔有机骨架 JUC-Z2 制备氮掺杂多孔碳材料. 与原始 JUC-Z2 相比, 制备的 多孔碳材料具有明显提高的气体吸附量和增强的吸附 焓. 其中优化的样品 JUC-Z2-900, 在 $273 \mathrm{~K}$ 下 $\mathrm{CO}_{2}$ 吸附 量高达 $113 \mathrm{~cm}^{3} \cdot \mathrm{g}^{-1}, \mathrm{CH}_{4}$ 吸附量高达 $60 \mathrm{~cm}^{3} \cdot \mathrm{g}^{-1}, 77 \mathrm{~K}$ 的 $\mathrm{H}_{2}$ 吸附量也达到 $246 \mathrm{~cm}^{3} \cdot \mathrm{g}^{-1}$, 超过了目前报道的大部 分多孔材料. 样品对于 $\mathrm{CO}_{2}$ 还表现出优异的选择性吸附 能力, JUC-Z2-900 的 $\mathrm{CO}_{2} / \mathrm{N}_{2}$ 的分离指数高达 $10, \mathrm{CO}_{2} / \mathrm{H}_{2}$ 的分离指数也高达 66 . 鉴于多孔有机骨架结构和组成 元素的多样性，本文为多孔碳材料的合成提供了一个新 路径.

\section{References}

[1] Figueroa, J. D.; Fout, T.; Plasynski, S.; Mcilvried, H.; Srivastava, R. D. Int. J. Greenh. Gas Con. 2008, $2,9$.

[2] Rochelle, G. T. Science 2009, 325, 1652

[3] Vallee, G.; Mougine, P.; Julian, S.; Furst, W. Ind. Eng. Chem. Res. 1999, 38, 3473.

[4] McKeown, N. B.; Makhseed, S.; Budd, P. M. Chem. Commun. 2002, 2780.

[5] McKeown, N. B.; Budd, P. M. Macromolecules 2010, 43, 5163.

[6] Kuhn, P.; Antonietti, M.; Thomas, A. Angew. Chem., Int. Ed. 2008, 47,3450 .

[7] Jiang, J.; Su, F.; Trewin, A.; Wood, C. D.; Campbell, N. L.; Niu, H.; Dckinson, C.; Ganin, A. Y.; Rosseinsky, M. J.; Khimyak, Y. Z.; Cooper, A. I. Angew. Chem. Int. Ed. 2007, 46, 8574.

[8] Côté, A. P.; Benin, A. I.; Ockwig, N. W.; O’Keeffe, M.; Matzger, A. J.; Yaghi, O. M. Science 2005, 310, 1166.

[9] El-Kaderi, H. M.; Hunt, J. R.; Mendoza-Cortés, J. L.; Côtés, A. P.; Taylor, R. E.; O'Keeffe, M.; Yaghi, O. M. Science 2007, 316, 268.

[10] Chen, L.; Honsho, Y.; Seki, S.; Jiang, D. J. Am. Chem. Soc. 2010, $132,6472$.

[11] Yuan, D.; Lu, W.; Zhao, D.; Zhou, H.-C. Adv. Mater. 2011, 23, 3723.

[12] Ben, T.; Qiu, S. CrystEngComm 2013, 15, 17.

[13] Pei, C.; Ben, T.; Qiu, S. Mater. Horiz. 2015, 2, 11.

[14] Peng, Y.; Ben, T.; Xu, J.; Xue, M.; Jing, X.; Deng, F.; Qiu, S.; Zhu, G. Dalton Trans. 2011, 40, 2720.

[15] Ren, H.; Ben, T.; Wang, E.; Jing, X.; Xue, M.; Liu, B.; Cui, Y.; Qiu, S.; Zhu, G. Chem. Commun. 2011, 46, 291.

[16] Zhang, T.; Wang, H.; Ma, H.; Sun, F.; Cui, X.; Zhu, G. Acta Chim. Sinica 2013, 71, 1598. (张婷婷, 王海涛, 马和平, 孙福兴, 崔小 强, 朱广山, 化学学报, 2013, 71, 1598.)

[17] Wang, W.; Yan, Z.; Yuan, Y.; Sun, F.; Zhao, M.; Ren, H.; Zhu, G. Acta Chim. Sinica 2014, 72, 557. (王维, 间卓君, 元野, 孙福兴, 赵明, 任浩, 朱广山, 化学学报, 2014, 72, 557.)

[18] Ben, T.; Ren, H.; Ma, S.; Cao, D.; Lan, J.; Jing, X.; Wang, W.; Xu, J.; Deng, F.; Simmons, J. M.; Qiu, S.; Zhu, G. Angew. Chem., Int. Ed. 2009, 48, 9457.

[19] Ben, T.; Pei, C.; Zhang, D.; Xu, J.; Deng, F.; Jing, X.; Qiu, S. Energy Environ. Sci. 2011, 4, 3991.

[20] Yuan, Y.; Sun, F.; Li, L.; Cui, P.; Zhu, G. Nat. Commun. 2014, 5, 4260.

[21] Lu, W.; Yuan, D.; Sculley, J.; Zhao, D.; Krisha, R.; Zhou, H.-C. J. 
Am. Chem. Soc. 2011, 133, 18126.

[22] Lu, W.; Sculley, J.; Yuan, D.; Krishna, R.; Wei, Z.; Zhou, H.-C. Angew. Chem. Int. Ed. 2012, 51, 7480.

[23] Konstas, K.; Taylor, J.; Thornton, A. W.; Doherty, C. M.; Lim, W.; Bastow, T. J.; Kennedy, D. F.; Wood, C. D.; Cox, B. J.; Hill, J. M.; Hill, A. J.; Hill, M. R. Angew. Chem. Int. Ed. 2012, 51, 6639.

[24] Ben, T.; Li, Y.; Zhu, L.; Zhang, D.; Cao, D.; Xiang, Z.; Yao, X.; Qiu, S. Energy Environ. Sci. 2012, 5, 8370.

[25] Xing, W.; Liu, C.; Zhou, Z.; Zhang, L.; Zhu, J.; Zhuo, S.; Yan, Z.; Gao, H.; Wang, G.; Qiao, S. Energy Environ. Sci. 2012, 5, 7323.

[26] Sevilla, M.; Parra, J. B.; Fuertes, A. B. ACS Appl. Mater. Interfaces 2013, 5, 6360.

[27] Seema, H.; Kemp, K. C.; Le, N. N.; Park, S.-W.; Chandra, V.; Lee, J.; Lim, K. S. Carbon 2014, 66, 320.

[28] Ben, T.; Shi, K.; Cui, Y.; Pei, C.; Zuo, Y.; Guo, H.; Zhang, D.; Xu, J.; Deng, F.; Tian, Z.; Qiu, S. J. Mater. Chem. 2011, 21, 18208.

[29] Zhang, L.; Su, Z.; Jiang, F.; Yang, L.; Qian, J.; Zhou, Y.; Li, W.;
Hong, M. Nanoscale 2014, 6, 6590.

[30] Zhao, X.; Zhao, H.; Zhang, T.; Yan, X.; Yuan, Y.; Zhang, H.; Zhao, D.; Zhu, G.; Yao, X. J. Mater. Chem. A 2014, 2, 11666.

[31] Guan, Y.; Ben, T.; Zhang, D.; Xu, J.; Pei, C.; Zhu, L.; Lu, C.; Meng, F.; Deng, F.; Qiu, S. Chem. J. Chinese Univ. 2012, 33, 2152. (关有 为, 贲腾, 张大梁, 徐君, 裴翠颖, 朱良奎, 逯春晶, 孟凡星, 邓 风, 雸式纶, 高等学校化学学报, 2012, 33, 2152.)

[32] Sumida, K.; Hill, M. R.; Horike, S.; Dailly, A.; Long, J. R. J. Am. Chem. Soc. 2009, 131, 15120.

[33] Loiseau, T.; Lecroq, L.; Volkringer, C.; Marrot, J.; Ferey, G.; Haouas, M.; Taulelle, F.; Bourrelly, S.; Llewellyn, P. L.; Latroche, M. J. Am. Chem. Soc. 2006, 128, 10223

[34] Ma, S.; Sun, D.; Simmons, J. M.; Collier, C. D.; Yuan, D.; Zhou, H.-C. J. Am. Chem. Soc. 2008, 130, 1012.

[35] Zhou, Z.; Xue, C.; Yang, Q.; Zhong, C. Acta Chim. Sinica 2009, 67 477. (周子娥, 薛春瑜, 阳庆元, 仲崇立, 化学学报, 2009, 67, 477.)

(Cheng, B.) 